\title{
Finding quantum effects in strong classical potentials
}

\author{
B. Manuel Hegelich ${ }^{1}$, Lance Labun ${ }^{1} \dagger$ and Ou Z. Labun ${ }^{1,2}$ \\ ${ }^{1}$ Department of Physics, University of Texas, Austin, Texas 78712, USA \\ ${ }^{2}$ Department of Physics, University of Arizona, Tucson, Arizona 85721, USA
}

(Received 15 July 2016; revised 11 April 2017; accepted 12 April 2017)

The long-standing challenge to describing charged particle dynamics in strong classical electromagnetic fields is how to incorporate classical radiation, classical radiation reaction and quantized photon emission into a consistent unified framework. The current, semiclassical methods to describe the dynamics of quantum particles in strong classical fields also provide the theoretical framework for fundamental questions in gravity and hadron-hadron collisions, including Hawking radiation, cosmological particle production and thermalization of particles created in heavy-ion collisions. However, as we show, these methods break down for highly relativistic particles propagating in strong fields. They must therefore be improved and adapted for the description of laser-plasma experiments that typically involve the acceleration of electrons. Theory developed from quantum electrodynamics, together with dedicated experimental efforts, offer the best controllable context to establish a robust, experimentally validated foundation for the fundamental theory of quantum effects in strong classical potentials.

Key words: intense particle beams, quantum plasma, strongly coupled plasmas

\section{Introduction: classical and quantum radiation}

Although the photoelectric effect showed that radiation is fundamentally a quantum process, long-wavelength $\left(\lambda \gg \lambda_{e}=\hbar / m_{e} c\right.$ where $\hbar=h / 2 \pi$ is the Plank constant divided by $2 \pi, m_{e}$ is the electron mass, and $c$ is the speed of light) radiation is equally well described by solving Maxwell's equations with a classical current as source. This classical approximation works because, in weak fields, the energy of the probe $E=h c / \lambda \ll m_{e} c^{2}$ is insufficient to excite the quantum structure of the electron. The electron Compton wavelength thus usually provides a natural length scale separating photons that should be treated as quantum $\left(\lambda \lesssim \lambda_{e}\right)$ and photons that can be treated as approximately classical $\left(\lambda \gg \lambda_{e}\right)$. However, by creating sufficiently strong fields, we can probe quantum dynamics with potential energy, rather than kinetic energy, with qualitatively different consequences.

The most remarkable and earliest recognized consequence is the discovery by Klein (1929) that when the potential difference exceeds the mass gap, $e \Delta V>2 m_{e}$ where $e$ is the elementary charge, the transmission coefficient for scattering is greater than unity.

$\dagger$ Email address for correspondence: labun@utexas.edu 
The potential emits particles at a rate that depends on the details of the potential but is always non-zero when $e \Delta V>2 m_{e} \cdot{ }^{1}$ This spontaneous particle creation was the first example of a non-perturbative effect in quantum theory, because it required solving the interaction with the potential to all orders, rather than expanding in a power series in the coupling $e$. Klein studied a step potential $V(z) \sim \Delta V \Theta(z)$, where $\Theta(z)$ is the Heaviside step function in which case the current, corresponding to pair creation, is proportional to the height of the potential step. In more realistic models, the potential depends on the space-time coordinates more smoothly, varying over a length scale greater than the electron Compton wavelength, $\lambda \gg \lambda_{e}$ and the rate of pair production is exponentially suppressed, proportional to $\exp \left(-\pi E_{c} /|\boldsymbol{E}|\right)$ where

$$
E_{c}=\frac{m_{e}^{2} c^{3}}{e \hbar}=1.32 \times 10^{18} \mathrm{~V} \mathrm{~m}^{-1},
$$

is known as the critical field and $|\boldsymbol{E}|$ is the local value of the electric field. Paradoxically, long-wavelength classical fields were found to generate short-wavelength $\sim \lambda_{e}$ radiation.

Klein's 'paradox' is the prototype to help understand phenomena and fundamental questions in domains other than electrodynamics. In quantum chromodynamics, the theory describing quark and gluon interactions inside hadrons, it is thought to help explain the high multiplicity and thermalization of particles created in the early stages of heavy-ion collisions (Andersson et al. 1983; Kharzeev, Levin \& Tuchin 2007; Gelis 2014; Gelis \& Tanji 2016). In gravity, the same physics underlies Hawking radiation (Brout et al. 1995; Kim 2007) and particle creation in the expanding universe (Anderson \& Mottola 2014a,b), both predictions subject to ongoing investigation and debate. Magnetic fields of the corresponding strength,

$$
B_{c}=\frac{1}{c} E_{c}=4.41 \times 10^{9} \mathrm{~T},
$$

are believed to exist around neutron stars (Harding \& Lai 2006). Even with such broad impact and long interest, spontaneous particle production has yet to be experimentally verified.

Strong fields break the classical approximation in another more subtle way. Already Lorentz noticed that conservation of momentum requires that the charged particle recoils from the radiation it emits according to Maxwell's equations, but that the recoil is not accounted for in the Lorentz force (Dirac 1938). This 'radiation reaction' problem can be perturbatively corrected by subtracting the lost momentum from the Lorentz force (Dirac 1938; Landau \& Lifshitz 1989), but even this solution is not complete: first, the perturbative approach breaks down when the momentum in the classical radiation is comparable to the momentum in the radiating electron. The onset of non-perturbative classical radiation approximately coincides with the condition that the field strengths in the electron rest frame are $\simeq E_{c}, B_{c}$ (Hadad et al. 2010), and in this same regime, high-energy quantized radiation becomes equally important (Ritus 1985). Second, the momentum absorbed by the electron is not removed from the accelerating field. Wheeler and Feynman solved the second problem by revising the Green's function to be the average of the retarded and advanced functions thus communicating the momentum absorbed by the electron back to the source of the field (Feynman 1966).

Feynman's propagator is a basic prescription of quantum field theory, and means radiation reaction is always consistently incorporated in quantum theory, but is not

\footnotetext{
${ }^{1}$ Klein obtained his result using the Dirac equation, but before antiparticles were understood or positrons discovered.
} 
compatible with classical radiation theory. In principle, instead of using the classical limit, one could simulate the full quantum dynamics as is done in lattice studies of quantum chromodynamics. In practice, such simulations are impossible for plasma systems of interest: to resolve the quantum dynamics, the simulation's grid spacing should be at least of the order of the electron Compton wavelength $\sim 10^{-12} \mathrm{~m}$ and the simulation volume should be large enough to incorporate tens to hundreds of plasma wavelengths, which is $\lambda_{p l} \simeq 10^{-6} \mathrm{~m}$, thus involving a space-time mesh of $\gtrsim\left(10^{7}\right)^{4}$ points. This wide separation of length scales is what ensures the classical limit remains an important tool to describe plasma physics even as quantum effects become important.

The challenge to describing particle dynamics and radiation in strong fields is thus to build a consistent and systematic framework to treat classical (long-wavelength) and quantized (short-wavelength) radiation where they are both important. The best controllable theoretical and experimental opportunities to explore these issues are in quantum electrodynamics (QED), facilitated by new ultra-high power lasers (Dunne 2009; Hegelich, Mourou \& Rafelski 2014). While spontaneous particle emission, requiring fields close to $10^{18} \mathrm{~V} \mathrm{~m}^{-1}$, is too rare an event to be measured in current laser facilities, we now study electron dynamics in moderately strong $\left(10^{14}-10^{15} \mathrm{~V} \mathrm{~m}^{-1}\right)$ fields to see the effect of radiation reaction and perturbative and non-perturbative quantum radiation processes that occur in the presence of the strong classical field (Di Piazza et al. 2012).

The current theory for these processes is founded upon the study of particle creation. Here we first review the methods in their original context of spontaneous particle creation and then critically examine their applicability to anticipated laser experiments. In this well-studied framework for QED in strong fields, average particle creation rates and light-by-light scattering involving low-energy photons $\left(\hbar \omega \ll m_{e} c^{2}\right)$ are consistently and accurately described because the long-wavelength fields do not resolve the short-wavelength quantum fluctuations. For electrons and high-energy photons with $\hbar \omega \gtrsim m_{e} c^{2}$, scattering events that involve energies of order the electron mass require a quantum description, because roughly speaking they probe the structure of the electron. For the field strengths in anticipated experiments on the Texas Petawatt $\left(I \simeq 3 \times 10^{22} \mathrm{~W} \mathrm{~cm}^{-2}\right.$, or $\left.|\boldsymbol{E}| \gtrsim 10^{15} \mathrm{~V} \mathrm{~m}^{-1}\right)$ and at the Extreme Light Infrastructure $\left(\gtrsim 10^{23} \mathrm{~W} \mathrm{~cm}^{-2}\right.$, or $|\boldsymbol{E}| \gtrsim 5 \times 10^{15} \mathrm{~V} \mathrm{~m}^{-1}$ ), such events are relatively rare, and one expects that classical dynamics suffices to determine evolution between events.

Our goal is the accurate and reliable prediction of high-intensity laser experiments, and we discuss here how to construct a consistent and systematic theory for the plasma dynamics, starting with single-particle radiation phenomena. As such, we consider the experimental range of single-particle conditions, rather than attempting to optimize the signal from the current, untested calculational approaches.

With this goal in mind, any description of the interactions between quantized particles requires a systematic investigation of the perturbation theory. Based on well-established features of quantum field theory especially involving massless gauge fields such as photons, we argue that QED in strong classical fields (laser 4-potentials $\left.\left|e A^{\mu}\right| \gg m_{e}\right)$ gives rise to $O(1)$ corrections to probabilities of events. We describe the impact of these corrections on predictions involving real high-energy electrons and photons and the contrast from low-energy vacuum phenomena (i.e. no quantized particles present) or non-relativistic electrodynamics. With its broader applications, a framework to describe the interactions of particles with strong, classical fields is an unsolved problem that would have fundamental impacts both within and beyond plasma physics. We introduce the concepts needed to improve on the current theory and how we expect the development of more complete and systematic theory to impact the broader questions in related fields arising from Klein's 'paradox'. 


\section{Spontaneous pair production and semiclassical methods}

Although Klein's calculation relied on a simple scalar step potential, it captured the essential physics. Shortly afterward, Sauter showed that particle emission occurs in a constant electric field, which with specific choice of gauge corresponds to a linear potential $A^{\mu}=\left(A_{0}, \mathbf{0}\right)$ with $A_{0}=-|\boldsymbol{E}| z$ (Sauter 1931). ${ }^{2}$ For a constant field of infinite extent, the potential difference is $2 m_{e}$ over a length $\Delta z=2 m_{e} /|e \boldsymbol{E}|$. Using intuition from the quantum mechanical barrier scattering problem, the mass gap $2 m_{e}$ between positive and negative frequency states implies a classically forbidden region with width $\Delta z$ in which the wavefunction of the electron decays exponentially. The decay length is proportional to the energy of the state and so is maximized for an electron at rest $\sim 1 / m_{e}$. Tunnelling through the barrier is therefore exponentially suppressed $\sim \mathrm{e}^{-m \Delta z}=$ $\mathrm{e}^{-2 m_{e}^{2} /|e \boldsymbol{E}|{ }^{3}}$ The exponent exhibits the critical field (1.1) which sets the scale for pair production to become an order-1 effect and therefore also estimates the breakdown scale of the theory.

To obtain their results, Klein and Sauter solve the Dirac equation (or Klein-Gordon equation for a scalar) in the presence of a c-number electromagnetic potential, $A_{c l}^{\mu}$,

$$
\left(\mathrm{i} \not \partial-e A_{c l}(x)-m\right) \psi(x)=0 .
$$

The subscript 'cl' stands for classical because the potential $A^{\mu}(x)$ is non-dynamical, and incorporating it into the solution of the Dirac equation assumes that the potential varies slowly relative to the dynamics of the quantized electron, i.e. the following condition is satisfied:

$$
\frac{\partial V(x)}{V(x)} \ll \frac{\partial \psi(x)}{\psi(x)} \Leftrightarrow \quad k_{c l}^{\mu} \ll p_{e}^{\mu} .
$$

Expanding each field in Fourier modes shows it is equivalent to the wavelength of the 'classical' modes being much larger than the wavelength of the quantized modes. In this case, a single photon of the classical field does not resolve the quantum structure of the electron. This condition is consistent with separately solving the classical electromagnetic field dynamics. As discussed below, it allows the equations of motion for the vacuum expectation value of the current (arising from spontaneously produced particles) and slowly varying electromagnetic fields to be closed and solved self-consistently at the long-wavelength scale. The approximation (2.1), (2.2) also provides a starting point to calculate rates of quantum processes compatible with classical laser-plasma simulations, but we shall discuss processes with electrons (and high-energy photons) in later sections.

Equation (2.2) is a necessary condition; it does not determine useful applications. The approximation (2.1), (2.2) is applied in cases that the potential is leading order. In contrast, standard perturbation theory assumes the particle is free at leading order and interactions are suppressed by a small coupling. In QED, that means solving the free Dirac equation $(\mathrm{i} \not \partial-m) \psi=0$ to obtain a basis of plane-wave solutions and adding interactions with photons as a perturbative expansion in $\alpha=\mathrm{e}^{2} / 4 \pi \hbar c$. However, if $e A^{\mu} \gtrsim p_{e}^{\mu}$, perturbation theory in $e A^{\mu}$ breaks down. Instead, the classical potential should be taken into account to all orders, summed into a new leading order by organizing perturbation theory as an expansion around solutions to (2.1).

\footnotetext{
${ }^{2}$ We use natural units, $\hbar=c=1$, restoring them explicitly where adding insight.

${ }^{3}$ One may get the exponent correct by massaging the argument further, but in general, beyond the relevant dimensionless ratios of parameters, such qualitative arguments will not reproduce order-1 prefactors.
} 
The solutions of (2.1) often display non-polynomial dependence on $e A_{c l}$, though they can be expanded in powers of $e A_{c l}$. For a plane-wave potential (discussed more extensively in the next section), the first terms in the expansions are explicitly verified to agree with a perturbative calculation of the electron scattering from the potential (Lavelle, McMullan \& Raddadi 2013).

Treating the electromagnetic field as classical, via (2.1), (2.2), is a necessary and consistent approximation scheme when the electron field is integrated out and only dynamics in vacuum is considered. The process of integrating out is equivalent to and often implemented by using the leading-order equation of motion for the relevant degrees of freedom to remove them from the (quantum) generating functional. Thus, solving (2.1) allows one to remove electrons as an explicit degree of freedom and determine the effect of electron fluctuations on the dynamics of long-wavelength electromagnetic fields.

However, as $|\boldsymbol{E}|=E_{c}$, the potential difference is $m_{e}$ over the Compton wavelength of the electron $\lambda_{e}=1 / m_{e}$, and the relevant time scale for pair creation approaches $\hbar / \alpha m_{e} c^{2}=1.8 \times 10^{-19} \mathrm{~s}$ (Labun \& Rafelski 2009), indicating that the field-particle dynamics is no longer adiabatic. Indeed, the rapid conversion of field energy to particles has been suggested to limit the electric field strength achievable (Bulanov et al. 2010; Fedotov et al. 2010). We will explain this breakdown more thoroughly below. Other than this emergent dynamics, treating the electromagnetic field as classical provides a good leading-order description of the pair creation dynamics due largely to the smallness of the electromagnetic coupling $\alpha=\mathrm{e}^{2} / 4 \pi \hbar c \simeq 1 / 137$.

This procedure of integrating out the electrons was first achieved by Heisenberg \& Euler (1936), who unified Sauter's investigation with the first calculations of light-by-light scattering (Euler \& Kockel 1935), by calculating the correction to the energy of a constant electromagnetic field due to quantum fluctuations of electron-positron pairs. In so doing, they obtained the first effective potential and first low-energy effective field theory, a framework of great importance that we will explain below. The Heisenberg-Euler effective potential shows that long-wavelength $\lambda \gg \lambda_{e}$ light-light interactions can be expanded in a power series with succeeding terms suppressed by the ratio $|\boldsymbol{E}| / E_{c}$ :

$$
V_{\text {eff }} \simeq \frac{\alpha}{\pi}|\boldsymbol{E}|^{2} \sum_{n=1}^{\infty} a_{n}\left(\frac{|\boldsymbol{E}|}{E_{c}}\right)^{2 n}
$$

(suitably generalized to be parity conserving and depend only on the field tensor $F^{\mu v}$ and its dual in the presence of magnetic fields). Clearly the power series fails to converge as $|\boldsymbol{E}| \rightarrow E_{c}$. This breakdown signals the onset of non-perturbative physics (Dunne \& Hall 1999; Labun \& Rafelski 2010), which in this case we already know is the probability of particle creation being near unity.

Schwinger (1951) advanced the calculational technology by obtaining the effective potential (2.3) in a gauge-invariant approach that also allowed a straightforward definition of its imaginary part

$$
\operatorname{Im} V_{\text {eff }}=\frac{\alpha}{2 \pi^{2}}|\boldsymbol{E}|^{2} \sum_{n=1}^{\infty} \frac{1}{n^{2}} \mathrm{e}^{-n \pi E_{c} /|\boldsymbol{E}|},
$$

$V_{\text {eff }}$ is complex due to the particle creation instability; to create the electric field we must do work to separate charges to $z \rightarrow \pm \infty$ and the field can be screened and reduced by creating electron-positron pairs. The imaginary part limits the radius of convergence of the power series (2.3) and ensures that the series is only asymptotic. 
Moreover, recalling this energy balance points to a small inconsistency in the framework: the electric field in the calculation is taken as prescribed, and the energy required to create the pair is not removed from the field. This can be effectively corrected by incorporating the pair creation dynamics into a local mean-field current $\left\langle j^{\mu}(x)\right\rangle$ that sources the electromagnetic field via Maxwell's equation

$$
\left\langle j^{\mu}(x)\right\rangle=\partial_{\nu} F^{\nu \mu}(x) .
$$

The vacuum expectation value of the current $\left\langle j^{\mu}\right\rangle$ is calculated under the approximation (2.1), (2.2) and evolved forward in time using (2.1) according the Schwinger-Keldysh formalism for non-equilibrium quantum dynamics (Cooper \& Mottola 1989; Kluger et al. 1992; Kluger, Mottola \& Eisenberg 1998). The resulting system of kinetic equations is closed and consistent at the long-wavelength scale $\lambda \gg \lambda_{e}$. The classical field and mean particle number dynamics can also be followed with greater temporal resolution using a real-time statistical formulation (Hebenstreit, Berges \& Gelfand 2013).

This system of equations is consistent as long as the time scale for a quantum event (pair production) is much smaller than the time scale for changes in the classical field, which is estimated by the plasma frequency

$$
\tau_{q} \ll \tau_{p l}=\frac{2 \pi}{\omega_{p l}} .
$$

Numerical study of spontaneous pair production dynamics indicates that $\tau_{q} \sim 1 / E_{p}$, where $E_{p}$ is the energy of the created particle (Kluger et al. 1998; Anderson \& Mottola 2014b). To estimate the plasma time scale, we consider near-critical fields creating pairs at a rate given by the first term in the series (2.4) (Cohen \& McGady 2008; Labun \& Rafelski 2009)

$$
\frac{\mathrm{d} N_{\text {pairs }}}{\mathrm{d}^{4} x}=\frac{\alpha}{8 \pi^{3}}|\boldsymbol{E}|^{2} \mathrm{e}^{-\pi E_{c} /|\boldsymbol{E}|} .
$$

Then using the classical definition of the plasma frequency for pairs created by the field, $\omega_{p l}^{2}=\mathrm{e}^{2} n_{e} / m_{e}$ with the density of charges $n_{e}=2 n_{\text {pairs }}=2 \tau_{p l}\left(\mathrm{~d} N_{\text {pairs }} / \mathrm{d}^{4} x\right)$, we obtain

$$
\tau_{p l}=\frac{1}{m_{e}}\left(\frac{2 \pi^{2}}{\alpha} \frac{m_{e}^{2}}{|e \boldsymbol{E}|}\right)^{2 / 3} \mathrm{e}^{\pi E_{c} / 3|\boldsymbol{E}|}
$$

Although crude, solving this estimate shows that (2.6) is satisfied by orders of magnitude even up to $|\boldsymbol{E}|=E_{c}$, due to the smallness of $\alpha$. At this level, the closed system of pair creation and the electron-positron plasma dynamics backreacting on the field appears to be consistently classical as desired.

A more insidious inconsistency takes over in the limit $|\boldsymbol{E}| \rightarrow E_{c}$ however. The conversion rate of field energy into rest mass $\mathrm{d}\left\langle u_{m}\right\rangle / \mathrm{d} t$ is characterized by a kinetic time scale, the 'materialization time' (Labun \& Rafelski 2009)

$$
\frac{1}{\tau_{\text {mat }}}=\frac{2}{|\boldsymbol{E}|^{2}} \frac{\mathrm{d}\left\langle u_{m}\right\rangle}{\mathrm{d} t} \simeq \frac{1}{\tau_{e}}|\boldsymbol{E}|^{2} \mathrm{e}^{-\pi E_{c} /|\boldsymbol{E}|} \quad \tau_{e}=\frac{\pi^{2}}{\alpha m_{e}} \simeq 1.7 \times 10^{-18} \mathrm{~s} .
$$

Already at $|\boldsymbol{E}| \sim 0.4 E_{c}$, the materialization time is less than 1 femtosecond, indicating that a significant fraction $(\sim 1 / e)$ of the laser energy would be converted into pairs in less than one period of an optical laser field (see also (Bulanov et al. 2010; Fedotov et al. 2010)). 
This implies $\sim 1 / e$ of the total system's energy is in the pair plasma, and therefore the plasma-induced electric and magnetic fields are similar in magnitude to the laser field and varying on the time and length scales of the plasma density

$$
\Delta t, \Delta L \sim\left(\frac{\mathrm{d} N_{\text {pairs }}}{\mathrm{d}^{3} x}\right)^{-1 / 3} \sim\left(\tau_{\text {mat }} \frac{\mathrm{d} N_{\text {pairs }}}{\mathrm{d}^{4} x}\right)^{-1 / 3} \sim 9 / m_{e},
$$

an estimate agreeing with the real-time simulations under the same approximation (Hebenstreit et al. 2013). Equation (2.10) shows that the mean distance between particles is much smaller than the classical electron radius $9 / m_{e} \ll 1 / \alpha m_{e} \simeq 137 / m_{e}$, and (2.9) thus estimates the field scale at which pair creation and field dynamics are strongly coupled to each other. Subsequent pair creation events are significantly affected by the presence of nearby charges, which indicates that the vacuum (no particles present) and slowly varying (2.2) conditions in the estimates of pair creation are violated. An additional condition for this method to describe the particle and field evolution in laser experiments is therefore that the materialization time is greater than one laser period, $\tau_{\text {mat }}>T_{\text {laser }}$.

To establish that the framework of (Cooper \& Mottola 1989; Kluger et al. 1992, 1998; Hebenstreit et al. 2013) is a good approximation scheme to describe spontaneous pair production, it only remains to check that quantum corrections are under control, i.e. the expansion in $\alpha$. The two-loop $\left(\alpha^{2}\right)$ contribution to pair production has been calculated explicitly, showing that it only becomes important at $E \sim 60 E_{c}$ (Ritus 1975; Fliegner et al. 1997; Reuter, Schmidt \& Schubert 1997). In sum, the framework is valid and can be self-consistently closed in moderately strong, but smaller than critical fields $|\boldsymbol{E}| \lesssim 0.4 E_{c}$. On the other hand, recall also that classical radiation emission also becomes non-perturbative (a leading-order correction to electron dynamics) at this field strength (Hadad et al. 2010). Thus, this method breaks down near the critical field, and a new theory is needed to describe dynamics at or above this field scale.

The same framework describes cosmological particle creation and backreaction on black holes due to Hawking radiation. In these cases, the classical potential is gravitational and the quantum dynamics is similarly obtained by solving for the wavefunctions in the presence of the potential. Backreaction is included by calculating the source current (there the energy-momentum tensor) in the same approximation scheme (2.1), (2.2) and plugging it back into the field equations, as in (2.5).

Recent progress has also been made on the statistics of particles produced, toward improving the understanding of thermalization and multiplicity of particles created in heavy-ion collisions. Here, the early stages of a collision between two heavy ions, usually $\mathrm{Au}$ and $\mathrm{Pb}$ nuclei, are modelled by considering that hard collisions between partons inside the nucleons are relatively rare but respond to the long-wavelength gluon fields sourced by partons in the other nucleus. Solving the classical equations of motion (analogues of Maxwell's equations for non-abelian theory) for these longwavelength gluon fields suggests the creation of extended colour fields in the aftermath of the collision (Andersson et al. 1983; Kharzeev et al. 2007; Gelis \& Tanji 2016) that can decay via spontaneous emission.

\section{High-intensity laser experiments}

So far, we have studied how a specific non-perturbative process, spontaneous pair production, is described in quantum field theory by approximating the strong electromagnetic field as classical. In this study, the initial state of the system 
contains no particles, and the subsequent evolution is only defined in the mean-field approximation, which means considering dynamics at the long-wavelength scale of the classical field and averaging over the charge density at shorter distance. We need a different theoretical framework to predict the dynamics of particles interacting with a strong classical field and the relevant experimental observables such as (final state) energies and radiation emitted by particles strong laser fields. Indeed, the same considerations apply to the single particle dynamics at the onset of pair creation, when only a few particles are present and are accelerated by the strong field to radiate and possibly go on to create more pairs, as suggested by Fedotov et al. (2010), Bulanov et al. (2010). To develop a useful theory however, we first discuss the conditions and measurable results of experiments.

Most experiments involve a high-intensity $\left(I>10^{21} \mathrm{~W} \mathrm{~cm}^{-2},|\boldsymbol{E}|>10^{13} \mathrm{~V} \mathrm{~m}^{-1}\right)$ laser pulse striking a solid or gas target at rest in the laboratory frame. Current laser systems achieve peak intensities of $10^{22} \mathrm{~W} \mathrm{~cm}^{-2}$, and planned facilities will reach $10^{23}-10^{24} \mathrm{~W} \mathrm{~cm}^{-2}$, still much smaller than the intensity corresponding to the critical field (1.1)

$$
I_{c}=\epsilon_{0} c E_{c}^{2}=1.29 \times 10^{29} \mathrm{~W} \mathrm{~cm}^{-2} .
$$

To maximize the field strength seen by electrons, the density of electrons is chosen to be above the critical density

$$
n_{e}>n_{c r}=\frac{m_{e} \omega_{\text {laser }}^{2}}{\mathrm{e}^{2}}
$$

obtained by setting the plasma frequency equal to the laser frequency. Setting $n_{e}>$ $n_{c r}$ ensures the laser frequency is below the low-frequency cutoff for electromagnetic waves that propagate in the plasma, and consequently a large fraction of laser energy is absorbed or reflected, creating electric and magnetic fields in the plasma of the same order of magnitude as the incident laser field. (Note that $n_{c r}$ is unrelated to the critical field strength, being purely classical with no $\hbar$.)

The experiments are equipped with detectors that typically measure the particle momentum in a small region of phase space. For example, a high-energy particle spectrometer may cover a significant range in one momentum component (e.g. along the beam axis) but have a small angular acceptance, with particles only less than a few milliradians from the beam axis entering the detector. On the other hand, detectors with larger angular acceptance are typically designed for the low-energy $\left(\hbar \omega \ll m_{e} c^{2}\right.$ ) classical radiation. This situation is understandable given the foregoing focus on particle acceleration schemes.

To study quantum radiation and particle production processes, we will need to adapt diagnostics in everyday use at high-energy particle colliders such as Jefferson Lab or KEK, not to mention larger facilities such as the Relativistic Heavy Ion Collider (RHIC) and the Large Hadron Collider (LHC). These detectors are suited to the measurement of high-energy particles in larger volumes of phase space and the extraction of rare signal events amid a much larger number of background particles, capabilities also important for seeking quantum effects in high-intensity laser experiments. However, their designs are less prepared for the high flux of radiation from a typical laser-plasma experiment. For example, for Texas Petawatt parameters, current models predict $\gtrsim 10^{10}$ photons with energies $\gtrsim 1 \mathrm{MeV}$ entering $20^{\circ}$ by $20^{\circ}$ solid angle around the beam axis and distributed in time over an interval less than 1 ps. This flux is orders of magnitude greater than tracked by the LHC detectors, which now handle $\gtrsim 100 \mathrm{~s}$ of particle tracks every $100 \mathrm{ps}$ (the crossing rate of bunches at the collision points). While particle density would be mitigated 
by larger distance to the detector (as will probably be necessary anticipating larger detector infrastructure), dedicated development of new detector systems will be a necessary component of future high-intensity laser experiments, especially those seeking quantum effects.

One experiment so far has provided proof of principle that high-energy particle detectors are useful to diagnose laser-particle interactions. The SLAC E-144 experiment successfully collided the linear accelerator's $46.6 \mathrm{GeV}$ electron beam with a moderate-intensity laser $\left(I \simeq 5 \times 10^{17} \mathrm{~W} \mathrm{~cm}^{-2}\right)$. They detected nonlinear Compton scattering $N \gamma+e \rightarrow \gamma+e$ and pair production, achieving agreement with predictions in the weakly nonlinear regime $N<10$, where the strong classical field is not yet dominant (Bula et al. 1996; Bamber et al. 1999).

\section{Processes and observables for electrons in strong laser fields}

Predictions are derived by two methods: (i) analytic calculations of single electron dynamics, utilizing the above-described approximation; and (ii) numerical particle-in-cell (PIC) simulations, implementing a reduced model of quantum emission processes and/or radiation reaction modified equations of motion. The two differ in several important aspects.

Analytic theory calculations are equipped to investigate quantum interferences in the amplitudes, which in principle could be engineered to enhance the rate for a specific process to occur. For example, a sequence of $N$ (identical) electric field pulses is predicted to increase the probability of spontaneous pair creation by $N^{2}$, analogous to $N$-slit diffraction (Akkermans \& Dunne 2012). However, such calculations require that the electromagnetic field is known at all points in space-time, and thus they typically exclude any dynamical plasma effects that affect the profile of the input laser, classical radiation and backreaction of the calculated processes.

PIC simulations achieve the complement: developed initially to predict the nonlinear laser-plasma dynamics, they include the backreaction on the classical laser field due to the collection motion and radiation by the plasma. Due to the computational expense however, PIC simulations typically use one simulation (quasi-)particle to represent $N \gg 1$ physical electrons or ions. They can be adapted to special case single-particle studies and take into account evolving particle multiplicities, such as in Elkina et al. (2011). However in general, PIC codes are not suited to incorporate the stochastic character of quantum processes and instead implement simplified versions. A common but important simplification is that photons are emitted only exactly collinear to the electron 3-momentum, so as to exclude stochastic momentum dispersion within a simulation quasi-particle. Recent work has suggested this simplification is reasonably accurate, provided one can average over a large number $\sim 10^{3}$ simulations (Harvey, Ilderton \& King 2015); however statistical uncertainties in the simulations (and experiments) remain unknown. Moreover, because the long-wavelength electromagnetic fields are solved on a grid, PIC simulations intrinsically introduce a new length scale, the grid cell size, that separates classical electromagnetic dynamics from any quantized photon emission. This separation is artificial and should be removed systematically using techniques described below.

Even so, one can learn about the dynamics by well-designed calculations with the laser field treated as prescribed, and we summarize a few relevant results here. Most recent results cited here rely on solutions to the Dirac equation (2.1) with a plane-wave type potential in the transverse gauge: $A_{c l}^{\mu}=A_{\perp}^{\mu}(t-z)$, the wave propagates in the $+\hat{z}$ direction, hence a function only of $t-z$, and its polarization is 
in the transverse $(x, y)$-plane. The solution is known as the Volkov solution (Wolkow 1935; Brown \& Kibble 1964) and is invariant only under a restricted class of gauge transformations (see Di Piazza (2014) for a generalization of the Volkov solution). The common procedure is then calculate QED diagrams with the classical field incorporated into the basis of electron states used to form matrix elements and electron correlation functions. Real processes have only been calculated at tree level, without loop corrections. The one-loop (order $\alpha$ ) self-energy and photon polarization tensors have been obtained (Ritus 1985); however, their divergent parts contribute renormalization and thus are visible only in higher-order corrections, which have not been studied, and the real processes represented by their imaginary parts are equivalently obtained from tree-level diagrams.

Toward the construction of a more systematic quantum theory, it is useful to show that this method reproduces properties of QED perturbation theory. One of the more important results is that low-momentum radiative corrections factorize and exponentiate (Dinu, Heinzl \& Ilderton 2012; Ilderton \& Torgrimsson 2013). The low-energy, low-intensity limit of the photon emission probabilities agree with the classical limit (Ritus 1985). Additionally, the Ward-Takahashi identity, ensuring gauge invariance of amplitudes involving electron loops constructed from Volkov states, holds (Meuren, Keitel \& Di Piazza 2013), and the optical theorem explicitly checked for the one-loop polarization function for photons (Meuren et al. 2015b). These results, including the analysis of the propagator (Lavelle et al. 2013), arise from the fact that the Volkov solution is a Wilson line: a gauge-covariant, path-ordered line integral that can be expanded into an infinite sum of couplings to the here classical gauge potential. To see this, note that the Volkov solution for an electron can be written

$$
\psi_{V}(x)=\sum_{p} W_{p} \mathrm{e}^{-\mathrm{i} p x} u_{p}
$$

where $u_{p}$ satisfies the free Dirac equation $(\not p-m) u_{p}=0$ and the prefactor satisfies (Ritus 1972)

$$
\left(\mathrm{i} \not \partial-e A_{c l}\right) W_{p} \hat{O}=W_{p} \mathrm{i} \not \partial \hat{O}
$$

for any operator $\hat{O}$. Thus, somewhat more formally, $W_{p}$ satisfies an operator

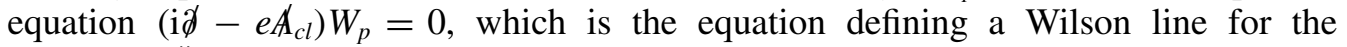
potential $A_{c l}^{\mu}$.

Beyond these field theoretic results, a small tome's worth of articles have been published predicting phenomenological signatures of high-energy photon emission and pair production, which we shall not review comprehensively here. In a monochromatic plane-wave field, momentum conservation requires that an electron can only radiate photons in integer multiples of the plane-wave wave vector, $k_{\text {out }}^{\mu}=N k_{\text {laser }}^{\mu} . N=1$ corresponds to perturbative Compton scattering, and $N>1$ is often called nonlinear Compton scattering because it requires absorbing $N$ quanta from the classical field and becomes more probable as the laser $a_{0} \gg 1$. Many studies focus on the kinematical consequences of short (few cycle) laser pulses, which broaden the momentum distribution of photons the electron can absorb (Di Piazza, Hatsagortsyan \& Keitel 2010; Heinzl, Seipt \& Kampfer 2010b; Mackenroth \& Di Piazza 2011, 2013; Seipt \& Kampfer 2011, 2012; Harvey et al. 2012). Pair conversion by photons and pair emission by electrons propagating in the field has been studied under similar conditions (Heinzl, Ilderton \& Marklund 2010a; Ilderton 2011; Nousch et al. 2012; 
Titov et al. 2012; King \& Ruhl 2013; Meuren et al. 2015a,b; Jansen \& Mller 2016). Even the effective neutrino-photon coupling and the axial anomaly have received attention (Shaisultanov 1998, 2000; Gies \& Shaisultanov 2000; Meuren, Keitel \& Di Piazza 2015c).

One phenomenologically important fact is that for $a_{0} \gg 1$ pair creation in a general plane-wave field is well approximated by convolving the local constant crossed field probability of creation with the classical dynamics of the electrons (Meuren, Keitel \& Di Piazza 2016). This outcome may be understood by noting that $a_{0}$ is the inverse of the Keldysh parameter (Keldysh 1965; Schutzhold, Gies \& Dunne 2008), which when $1 / a_{0} \sim 1$ roughly indicates that the frequency of the classical field is important. Conversely when $1 / a_{0} \ll 1$, the probability approaches the constant field result. Thus, for $a_{0} \gg 1$ laser fields, varying only in one lightcone direction, the process is local: there are no nonlinear vacuum polarization effects in a single plane wave of arbitrary spectral composition (Schwinger 1951) (a second wave vector must be introduced to break the symmetry), and pair creation in a light-like electric field is a local event (Ilderton, Torgrimsson \& Wårdh 2015). This property is particularly important for constructing a systematic quantum theory; if it were not true, we would have to worry about the length scale over which non-local correlations in the field could impact short-wavelength (quantum) dynamics.

\section{Effective field theory for strong field processes}

With the goal of constructing a predictive theory, what is the salient difference between pair creation in vacuum and electron dynamics in strong fields? In the study of spontaneous pair creation or light-by-light scattering with no real particles present, there are two length scales: the wavelength of variation of the classical field and the Compton wavelength of the electron. The condition (2.2) is the statement that these length scales are widely separated. Physically, the electron is a heavy particle, whose fluctuations are point-like relative to the variation of the (classical) field. The electron can therefore be 'integrated out', that is, the degrees of freedom can be removed from the theory as fluctuating too quickly to be resolved by the long-wavelength dynamics.

Integrating out the electron field to one-loop order yields the Heisenberg-Euler effective potential for long-wavelength $\left(\lambda \gg \lambda_{e}\right)$ electromagnetic fields, which is thus an expansion in both the ratio $\omega / m_{e}$, controlling the importance of derivative corrections and the QED coupling $\alpha$, controlling the importance of additional loop corrections (see (Ritus 1975) for the two-loop action). Although a complex analytic expression is known to all orders in the fields, applying the effective potential to any real process for electromagnetic fields in vacuum utilizes the expansion in powers of $1 / m_{e}^{4}$ (the fourth power due to the fact that only field invariants $\sim F^{2}$ can appear), shown in (2.3). As described above, the expansion in powers of $1 / m_{e}^{4} \sim 1 / E_{c}^{2}$ breaks down at the critical field coinciding with the onset of significant pair creation - which anyway breaks the no-particles-present condition. ${ }^{4}$

In contrast, processes involving real high-energy $\hbar \omega \gtrsim m_{e} c^{2}$ photons or electrons in the classical field involve at least one additional length scale, the de Broglie wavelength of the particle. It is simpler from now on to refer to momentum scales

\footnotetext{
${ }^{4}$ The first two terms in the power series $n=2,3$ are a very good approximation up to $|\boldsymbol{E}| \simeq E_{c}$, and the divergence of the power series manifestly coincides with the imaginary part becoming order 1 (Labun \& Rafelski 2010). Pure magnetic fields are stable, suggesting one might apply the Heisenberg-Euler effective potential to nonlinear dynamics of constant magnetic fields with magnitude greater than $B_{c}$. However the dynamics obviously introduces time derivatives on the fields, and the likely application (magnetized compact object atmospheres) involves photons and plasmas with particles in the keV energy range.
} 
$p, k \sim \hbar c / L$. For photons with momentum $k \ll m_{e}$, electrons are again heavy and can be integrated out. The Euler-Heisenberg effective potential thus suffices to calculate probabilities for vacuum birefringence, photon splitting and four-wave mixing. Photons with momentum $k \sim m_{e}$ and low-momentum electrons however resolve fluctuations around the scale $m_{e}$ and a full quantum theory with dynamical electron and photon degrees of freedom is necessary.

The case of greatest interest phenomenologically is photons and electrons with momentum $k, p \gg m_{e}$, as arise in the final state of a typical acceleration experiment. In this case, there is a large hierarchy of momentum scales

$$
E+p_{z} \gg\left|e A_{c l}^{\mu}\right| \gg m_{e} \gg \omega_{\text {laser }}
$$

where $e A_{c l}$ is the amplitude of the classical laser potential. The use of $E+p_{z}=p_{+}$the lightcone momentum conjugate to the lightcone coordinate $x_{-}=t-z$ is natural due to the plane-wave symmetry of the background. Current high-intensity laser systems on which the next experiments will be conducted have

$$
a_{0}=\frac{\left|e A_{c l}^{\mu}\right|}{m_{e}} \sim 10-350
$$

at their peak. ${ }^{5}$

This hierarchical separation of momentum or length scales is important to account for in quantum processes, because perturbation theory generically introduces corrections that are parametrically large, involving logarithms of ratios of the physical scales. Although the coupling constant is small for QED, a widely separated hierarchy, such as $E+p_{z} \sim 10^{3} m_{e} \sim 10^{8} \omega_{\text {laser }}$, enhances quantum corrections to becoming relevant. As an example, we consider the radiative corrections to the rate of photon emission. The largest difference between the tree-level and one-loop rates is a double logarithm involving the scales seen in (5.1) (Labun \& Zhang 2017)

$$
\frac{\Gamma^{(1-\text { loop })}(e \rightarrow e \gamma)}{\Gamma^{(\text {tree })}(e \rightarrow e \gamma)} \underset{-q^{2} \gg m_{e}^{2}}{\simeq} 1-\frac{\alpha}{4 \pi} \ln \frac{-q^{2}}{m_{e}^{2}} \ln \frac{-q^{2}}{E_{d}^{2}},
$$

where $-q^{2}=-\left(p-p^{\prime}\right)^{2}$ is the squared 4-momentum change by the electron. The coefficient is obtained by evaluating the one-loop correction to the electron-photon vertex shown in figure 1, with the laser field included to all orders by using the dressed propagator for the electron.

One can guess the form of (5.3) realizing that, even in the presence of a strong classical field, the short-distance behaviour of the amplitude must reproduce zero-field $\left(A_{c l}^{\mu} \rightarrow 0\right)$ QED. Fortunately, amplitudes for QED in classical laser fields do have the same short-distance behaviour as in zero-field QED. ${ }^{6}$ The classical field affects only the long-distance dependence, here encoded in $E_{d}$.

The momentum scale $E_{d}$ is the energy resolution of a detector and is an infrared cutoff distinguishing radiation from the electron (Peskin \& Schroeder 1995); in other words it measures the precision to which the electron momentum can be known. Here $E_{d}$ is set by the classical radiation, which having $\omega_{c l . r a d} \ll m_{e}$ is a continuous process

${ }^{5}$ The vector potential $A^{\mu}$ is well defined for a high-intensity field, because both the field intensity $|\boldsymbol{E}|^{2}$ and Fourier decomposition (distribution in $k$-space) can be measured with precision smaller than the absolute values. Consequently, the canonical degrees of freedom $A^{\mu}(x), \partial_{t} A^{\mu}(x)$ commute to very good approximation, the field is classical, the photon occupation number is not fixed.

${ }^{6}$ These features were not made manifest in the computation by (Morozov, Narozhnyi \& Ritus 1981), which focussed on the asymptotics for $\chi \gg 1$, and will elaborated by us in a dedicated publication. 


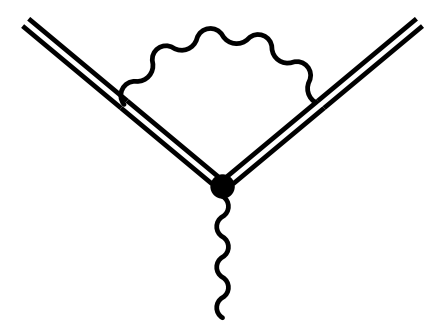

FIGURE 1. One-loop correction to the electron-photon vertex. The double line indicates the dressed propagator, which incorporates the classical potential to all orders. Details of the calculation will be presented elsewhere.

relative to photon emission. We therefore estimate this scale as the cyclotron frequency of the electron in the laser field $\omega_{\text {cl.rad. }} \sim \omega_{c y c}=|e \boldsymbol{B}| / p$. This can be written Lorentz invariantly by going to the instantaneous rest frame of the electron, where the field strength is $\left|\boldsymbol{B}^{\prime}\right|$ and

$$
E_{d} \sim \omega_{c y c}=\frac{\left|e \boldsymbol{B}^{\prime}\right|}{m_{e}}=\chi,
$$

with

$$
\chi^{2}=\frac{1}{m_{e}^{4}} p_{\mu} e F^{\mu v} e F_{\nu \lambda} p^{\lambda}=e^{2}\left(p \cdot P_{\text {laser }}\right)
$$

the Lorentz invariant presenting the centre of mass energy in the collision between the electron and the classical field (the momentum density of the field is multiplied by the Compton volume of the electron $\lambda_{e}^{3}=1 / \mathrm{m}_{e}^{3}$ to form a momentum). The dimensionless $\chi / m_{e}$ controls the magnitude of quantum effects, as shown by explicit calculation, and the limit $\chi / m_{e} \rightarrow 1$ implies the electric field seen by the electron in its rest frame is equal to the critical field (1.1).

Equation (5.3) represents perturbative corrections to the emission probability that are present both in zero-field QED and QED with a classical laser potential. The role of the laser field is to make single-photon emission, the tree-level amplitude, possible. The physical reason for the size of these corrections is the widely separated hierarchy of scales (5.1) and will arise under any consideration of high-energy electrons recoiling with $-q^{2} \gtrsim m_{e}^{2}$. Intuitively, one can think of the associated emission (or absorption) of a large number of lower-energy photons, as shown in figure 2 . The energy of these lower-energy photons can lie anywhere between the low-energy region $E_{d} \sim \chi$ and the electron's recoil, which encompasses a large region of phase space when $-q^{2} \ggg \chi^{2}$. These lower-energy photons are distinguishable from classical field, which in most applications, using the quasi-constant approximations, consists of only the zero mode; indeed photons at $E_{d}$ momentum scale coincide with the classical radiation predicted by the Lorentz force.

PIC simulations necessarily introduce another momentum scale, the inverse of the grid spacing, which sets an upper cutoff on the frequency of classical electromagnetic fields solved on the grid. For accurate single electron trajectories, this momentum scale should be chosen high enough to resolve the cyclotron frequency of electrons in the laser field (Gonoskov et al. 2015; Arefiev et al. 2016), and thus it naturally 


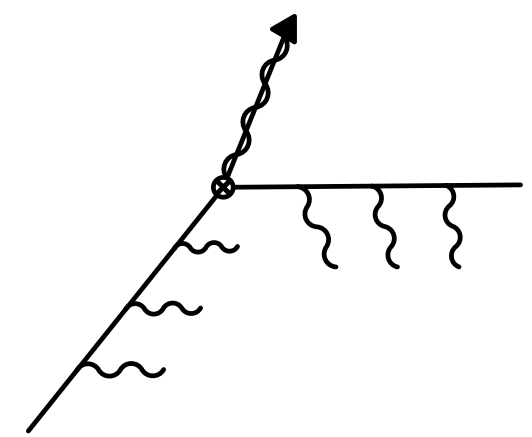

FIGURE 2. Real radiative corrections that correspond to the large logarithm (5.3), that is the emission of (any number of) lower-energy photons between the laser field frequency and the energy of the detected high-energy photon.

separates the classical and quantum radiation processes. The quantum emission rates should be evaluated with explicit knowledge of this scale, which is only possible with the methods we have briefly introduced in this article.

This does not require the fractional change in the electron momentum to be large: for a 50-MeV electron co-propagating with $a_{0} \sim 10^{2}$ laser, $\chi / m_{e} \sim 10^{-6}$, emitting a $5 \mathrm{MeV}$ photon is subject to a $\sim 6-15 \%$ correction. The emission probability for a photon with energy $k^{\mu} \sim 50 \mathrm{MeV}$ (still only one per cent of the electron's classical kinematic momentum $\sim a_{0}^{2} m_{e}$ ) is corrected by nearly $50 \%$. Since photons emitted in the energy range $k^{\mu} \sim 500 \mathrm{keV}-50 \mathrm{MeV}$ are a primary observable of interest in laser experiments, and simulations with the current PIC models suggest they are produced in observable numbers, it is necessary to ensure predictions of the photon emission spectrum and angular distribution are correct in this region. Corrections of the magnitude suggested (5.3) are more than enough to perturb the photons from their expected locations in the experimental apparatus and miss the (typically small-aperture) detectors completely. We are concerned with being able to verify predictions of QED effects: a detector merely 'seeing something' does nothing to validate the theory.

For an electron with $\gtrsim 50 \mathrm{MeV}$ energy co-propagating with the laser, the field in its rest frame is reduced by the Lorentz factor, and $\chi \sim 10^{-5}$. Then (5.3) implies that the emission probability for a photon with similar energy $k^{\mu} \sim 50 \mathrm{MeV}$ is corrected by nearly $50 \%$. For a moderate-energy photon $k^{\mu} \sim m_{e}$, the first logarithm in (5.3) is replaced by a number of order 1 , and the correction is $2 \%-5 \%$, depending on the electron momentum. Note that this is equal or larger than corrections implied by classical models of radiation reaction-corrected dynamics (Hadad et al. 2010).

\section{Conclusions}

Finding quantum effects in strong fields will require substantial combined theoretical and experimental efforts. Here, we have only touched briefly on the supporting computational infrastructure, that is necessary to connect the theory, describing high-energy, short-distance quantum dynamics, to the experiments, involving low-energy, long-distance classical dynamics. However, it is clear that more complete, systematic method to relate quantum and classical dynamics, must be incorporated into the numerical simulations that are used to interpret laser-plasma experiments. 
We have provided a preview of results proving that additional theory, beyond the tree-level amplitudes with the laser field treated as non-dynamical, is required to accurately describe quantum radiation processes by electrons in strong fields. Moreover, these same calculational methods underlie several outstanding issues in theoretical physics, from particle production in heavy-ion collisions to Hawking radiation in black holes, highlighting the necessity of a more complete theory of quantum and classical radiation and the transition between. As physics relies on experimental verification, it is of pressing importance to develop the theory in parallel with ongoing high-intensity laser experiments, so as to provide predictions of directly relevant experimental observables. To this end, we anticipate that the experience offered by high-energy hadron collisions will be invaluable, both in designing and building detectors and in defining observables to help test the theory.

Concerted, coordinated effort in this program will not only address some of the longest-standing problems in plasma physics (radiation reaction and spontaneous pair production) but also provide the first experimental foundation for fundamental theoretical issues in gravity and strong nuclear interactions.

\section{Acknowledgements}

B.M.H. and L.L. are supported by the Air Force Office of Scientific Research (FA9550-14-1-0045) as well as NNSA cooperative agreement de-na0002008, the

Defense Advanced Research Projects Agencys PULSE program (12-63-PULSEFP014), and the National Institutes of Health SBIR_1_LPT_001.

\section{REFERENCES}

AkKermans, E. \& Dunne, G. V. 2012 Ramsey fringes and time-domain multiple-slit interference from vacuum. Phys. Rev. Lett. 108, 030401.

Anderson, P. R. \& Mottola, E. $2014 a$ Instability of global de Sitter space to particle creation. Phys. Rev. D 89, 104038.

Anderson, P. R. \& MotTola, E. $2014 b$ Quantum vacuum instability of eternal de Sitter space. Phys. Rev. D 89, 104039.

Andersson, B., Gustafson, G., Ingelman, G. \& Suostrand, T. 1983 Parton fragmentation and string dynamics. Phys. Rep. 97, 31-145.

Arefiev, A. V., Cochran, G. E., Schumacher, D. W., Robinson, A. P. L. \& Chen, G. 2016 Criterion for correctly simulating relativistic electron motion in a high-intensity laser field. AIP Conf. Proc. 1777, 050001.

Bamber, C., Boege, S. J., Koffas, T., Kotseroglou, T., Melissinos, A. C., Meyerhofer, D. D., Reis, D. A., RagG, W., Bula, C., McDonald, K. T. et al. 1999 Studies of nonlinear QED in collisions of $46.6 \mathrm{GeV}$ electrons with intense laser pulses. Phys. Rev. D 60, 092004.

Brout, R., Massar, S., Parentani, R. \& Spindel, P. 1995 A Primer for black hole quantum physics. Phys. Rep. 260, 329-454.

Brown, L. S. \& KibBle, T. W. B. 1964 Interaction of intense laser beams with electrons. Phys. Rev. 133, A705-A719.

BulA, C. et al. 1996 Observation of nonlinear effects in Compton scattering. Phys. Rev. Lett. 76, 3116-3119.

Bulanov, S. S., Esirkepov, T. Z., Thomas, A. G. R., Koga, J. K. \& Bulanov, S. V. 2010 On the Schwinger limit attainability with extreme power lasers. Phys. Rev. Lett. 105, 220407.

Cohen, T. D. \& MCGAdy, D. A. 2008 The Schwinger mechanism revisited. Phys. Rev. D 78, 036008 . 
Cooper, F. \& Mottola, E. 1989 Quantum back reaction in scalar QED as an initial value problem. Phys. Rev. D 40, 456-464.

Di PIAZZA, A. 2014 Ultrarelativistic electron states in a general background electromagnetic field. Phys. Rev. Lett. 113, 040402.

Di Piazza, A., Hatsagortsyan, K. Z. \& Keitel, C. H. 2010 Quantum radiation reaction effects in multiphoton Compton scattering. Phys. Rev. Lett. 105, 220403.

Di Piazza, A., Muller, C., Hatsagortsyan, K. Z. \& Keitel, C. H. 2012 Extremely highintensity laser interactions with fundamental quantum systems. Rev. Mod. Phys. 84, 1177-1228, arXiv:1111.3886.

Dinu, V., Heinzl, T. \& Ilderton, A. 2012 Infra-red divergences in plane wave backgrounds. Phys. Rev. D 86, 085037.

Dirac, P. A. M. 1938 Classical theory of radiating electrons. Proc. R. Soc. Lond. A 167, 148-169.

DunNe, G. V. 2009 New strong-field QED effects at ELI: nonperturbative vacuum pair production. Eur. Phys. J. D 55, 327-340.

Dunne, G. V. \& Hall, T. M. 1999 Borel summation of the derivative expansion and effective actions. Phys. Rev. D 60, 065002.

Elkina, N. V., Fedotov, A. M., Kostyukov, I. Y., Legkov, M. V., Narozhny, N. B., Nerush, E. N. \& RUHL, H. 2011 QED cascades induced by circularly polarized laser fields. Phys. Rev. ST Accel. Beams 14, 054401.

Euler, H. \& Kockel, B. 1935 Ueber die Streuung von Licht an Licht nach der Diracschen Theorie. Naturwissenschaft 23, 246-247.

Fedotov, A. M., Narozhny, N. B., Mourou, G. \& Korn, G. 2010 Limitations on the attainable intensity of high power lasers. Phys. Rev. Lett. 105, 080402.

FEYnMan, R. P. 1966 The development of the space-time view of quantum electrodynamics. Science 153 (3737), 699-708 (World Sci. Ser. 20th Cent. Phys. 27, 9 (2000)).

Fliegner, D., Reuter, M., Schmidt, M. G. \& Schubert, C. 1997 The two loop Euler-Heisenberg Lagrangian in dimensional renormalization. Theor. Math. Phys. 113, 1442-1451 (Teor. Mat. Fiz. 113, 289 (1997)), arXiv:hep-th/9704194.

Gelis, F. 2014 The initial stages of heavy ion collisions. Acta Phys. Polon. B 45 (12), 2257-2306.

Gelis, F. \& Tanji, N. 2016 Schwinger mechanism revisited. Prog. Part. Nucl. Phys. 87, 1-49.

Gies, H. \& Shaisultanov, R. 2000 On the axial current in an electromagnetic field and low-energy neutrino - photon interactions. Phys. Rev. D 62, 073003.

Gonoskov, A., Bastrakov, S., Efimenko, E., Ilderton, A., Marklund, M., Meyerov, I., Muraviev, A., Sergeev, A., Surmin, I. \& Wallin, E. 2015 Extended particle-in-cell schemes for physics in ultrastrong laser fields: review and developments. Phys. Rev. E 92 (2), 023305.

Hadad, Y., Labun, L., Rafelski, J., Elkina, N., Klier, C. \& Ruhl, H. 2010 Effects of radiation-reaction in relativistic laser acceleration. Phys. Rev. D 82, 096012.

Harding, A. K. \& Lai, D. 2006 Physics of strongly magnetized neutron stars. Rep. Prog. Phys. 69, 2631-2708.

Harvey, C., Heinzl, T., Ilderton, A. \& Marklund, M. 2012 Intensity-dependent electron mass shift in a laser field: existence, universality, and detection. Phys. Rev. Lett. 109, 100402.

Harvey, C. N., Ilderton, A. \& King, B. 2015 Testing numerical implementations of strong field electrodynamics. Phys. Rev. A 91 (1), 013822.

Hebenstreit, F., Berges, J. \& Gelfand, D. 2013 Simulating fermion production in $1+1$ dimensional QED. Phys. Rev. D 87 (10), 105006.

Hegelich, B. M., Mourou, G. \& Rafelski, J. 2014 Probing the quantum vacuum with ultra intense laser pulses. Eur. Phys. J. ST 223 (6), 1093-1104.

Heinzl, T., Ilderton, A. \& MARKLUnd, M. $2010 a$ Finite size effects in stimulated laser pair production. Phys. Lett. B 692, 250-256.

Heinzl, T., Seipt, D. \& KAMPfER, B. $2010 b$ Beam-shape effects in nonlinear Compton and Thomson scattering. Phys. Rev. A 81, 022125. 
Heisenberg, W. \& Euler, H. 1936 Consequences of Dirac's theory of positrons. Z. Phys. 98, $714-732$.

Ilderton, A. 2011 Trident pair production in strong laser pulses. Phys. Rev. Lett. 106, 020404.

ILDERTON, A. \& TORgRimsson, G. 2013 Scattering in plane-wave backgrounds: infra-red effects and pole structure. Phys. Rev. D 87, 085040.

ILDERTON, A., TORGRIMSSON, G.\& WARDH, J. 2015 Nonperturbative pair production in interpolating fields. Phys. Rev. D 92 (6), 065001.

JAnsen, M. J. A. \& MlleR, C. 2016 Strong-field breit-wheeler pair production in short laser pulses: identifying multiphoton interference and carrier-envelope-phase effects. Phys. Rev. D 93 (5), 053011.

Keldysh, L. V. 1965 Diagram technique for nonequilibrium processes. Sov. Phys. JETP 20, $1018-1030$.

Kharzeev, D., Levin, E. \& Tuchin, K. 2007 Multi-particle production and thermalization in high-energy QCD. Phys. Rev. C 75, 044903.

KIM, S. P. 2007 Hawking radiation as quantum tunneling in Rindler coordinate. JHEP 11, 048.

KING, B. \& RUHL, H. 2013 Trident pair production in a constant crossed field. Phys. Rev. D 88 (1), 013005.

KLEIN, O. 1929 Die Reflexion von Elektronen an einem Potentialsprung nach der relativistischen Dynamik von Dirac. Z. Phys. 53, 157-165.

Kluger, Y., Eisenberg, J. M., Svetitsky, B., Cooper, F. \& Mottola, E. 1992 Fermion pair production in a strong electric field. Phys. Rev. D 45, 4659-4671.

Kluger, Y., Mottola, E. \& EisenberG, J. M. 1998 The quantum Vlasov equation and its Markov limit. Phys. Rev. D 58, 125015.

LABUn, L. \& RAfelski, J. 2009 Vacuum decay time in strong external fields. Phys. Rev. D 79, 057901 .

LAbun, L. \& RAfelski, J. 2010 Dark energy simulacrum in nonlinear electrodynamics. Phys. Rev. D 81, 065026.

LABUN, L. \& ZHANG, O. 2017 (in preparation).

Landau, L. D. \& Lifshitz, E. M. 1989 The Classical Theory of Fields, 4th edn. 481p. Pergamon.

Lavelle, M., McMullan, D. \& Raddadi, M. 2013 Propagation in an intense background. Phys. Rev. D 87 (8), 085024.

Mackenroth, F. \& Di Piazza, A. 2011 Nonlinear Compton scattering in ultra-short laser pulses. Phys. Rev. A 83, 032106.

Mackenroth, F. \& Di PiazzA, A. 2013 Nonlinear double Compton scattering in the ultrarelativistic quantum regime. Phys. Rev. Lett. 110 (7), 070402.

Meuren, S., Hatsagortsyan, K. Z., Keitel, C. H. \& Di Piazza, A. $2015 a$ High-energy recollision processes of laser-generated electron-positron pairs. Phys. Rev. Lett. 114 (14), 143201.

Meuren, S., Hatsagortsyan, K. Z., Keitel, C. H. \& Di Piazza, A. $2015 b$ Polarization operator approach to pair creation in short laser pulses. Phys. Rev. D 91 (1), 013009.

Meuren, S., Keitel, C. H. \& Di PiazzA, A. 2013 Polarization operator for plane-wave background fields. Phys. Rev. D 88 (1), 013007.

Meuren, S., Keitel, C. H. \& Di Piazza, A. 2015c Nonlinear neutrino-photon interactions inside strong laser pulses. JHEP 06, 127, arXiv:1504.02722.

Meuren, S., Keitel, C. H. \& Di Piazza, A. 2016 Semiclassical picture for electron-positron photoproduction in strong laser fields. Phys. Rev. D 93 (8), 085028.

Morozov, D. A., NAROzhnYi, N. B. \& Ritus, V. I. 1981 Vertex function of electron in a constant electromagnetic field. Sov. Phys. JETP 53, 1103-1130.

Nousch, T., Seipt, D., KAMPfer, B. \& Titov, A. I. 2012 Pair production in short laser pulses near threshold. Phys. Lett. B 715, 246-250.

Peskin, M. E. \& Schroeder, D. V. 1995 An Introduction to Quantum Field Theory. AddisonWesley.

Reuter, M., Schmidt, M. G. \& Schubert, C. 1997 Constant external fields in gauge theory and the spin $0,1 / 2,1$ path integrals. Ann. Phys. 259, 313-365. 
Ritus, V. I. 1972 Radiative corrections in quantum electrodynamics with intense field and their analytical properties. Ann. Phys. 69, 555.

Ritus, V. I. 1975 The Lagrange function of an intensive electromagnetic field and quantum electrodynamics at short distances. Sov. Phys. JETP 42, 774-813.

RITUS, V. I. 1985 Quantum effects of the interaction of elementary particles with an intense electromagnetic field. J. Russ. Laser Res. 6, 497.

SAUTER, F. 1931 Uber das Verhalten eines Elektrons im homogenen elektrischen Feld nach der relativistischen Theorie Diracs. Z. Phys. 69, 742-764.

Schutzhold, R., Gies, H. \& Dunne, G. 2008 Dynamically assisted Schwinger mechanism. Phys. Rev. Lett. 101, 130404.

SCHWInger, J. S. 1951 On gauge invariance and vacuum polarization. Phys. Rev. 82, 664-679.

SEIPT, D. \& KAMPFER, B. 2011 Non-linear compton scattering of ultrashort and ultraintense laser pulses. Phys. Rev. A 83, 022101.

Seipt, D. \& KAmpfer, B. 2012 Two-photon Compton process in pulsed intense laser fields. Phys. Rev. D 85, 101701.

Shaisultanov, R. 1998 Photon neutrino interactions in magnetic field. Phys. Rev. Lett. 80, $1586-1587$.

Shaisultanov, R. 2000 The neutrino neutrino gamma amplitude in an external homogeneous electromagnetic field. Phys. Rev. D 62, 113005.

Titov, A. I., TAKabe, H., Kampfer, B. \& HosaKa, A. 2012 Enhanced subthreshold electronpositron production in short laser pulses. Phys. Rev. Lett. 108, 240406.

Wolkow, D. M. 1935 Uber eine Klasse von Losungen der Diracschen Gleichung. Z. Phys. 94, 250-260. 